International Workshop

"Stochastic Programming for Implementation and Advanced Applications"

(STOPROG-2012)

July 3-6, 2012, Neringa, Lithuania
ISBN 978-609-95241-4-6

L. Sakalauskas, A. Tomasgard, S. W.Wallace (Eds.):

Proceedings. Vilnius, 2012, pp. 78-82

(C) The Association of Lithuanian Serials,

Lithuania, 2012

doi:10.5200/stoprog.2012.14

\title{
MEASURES OF INFORMATION IN MULTISTAGE STOCHASTIC PROGRAMMINGa)
}

\author{
Franceska Maggioni ${ }^{1}$, Elisabetta Allevi ${ }^{2}$, Marida Bertocchi ${ }^{3}$ \\ ${ }^{1,3}$ Department of Mathematics, Statistic, Computer Science and Applications, Bergamo University, \\ Via dei Caniana 2, 24127 Bergamo ITALY \\ ${ }^{2}$ Department of Quantitative Methods, Brescia University, Contrada S. Chiara 50, 25122 Brescia ITALY \\ E-mails: ${ }^{2}$ francesca.maggioni@unibg.it; ${ }^{2}$ allevi@eco.unibs.it; ${ }^{3}$ marida.bertocchi@unibg.it
}

\begin{abstract}
Multistage stochastic programs, which involve sequences of decisions over time, are usually hard to solve in realistically sized problems. Providing bounds for their optimal solution may help in evaluating whether it is worth the additional computations for the stochastic program versus simplified approaches. In this paper we present a summary of the results in [22] where we generalize the value of information gained from deterministic, pair solution and rolling-horizon approximation in the two-stage case to the multistage stochastic formulation. Numerical results on a case study related to a simple transportation problem illustrate the described relationships.
\end{abstract}

Keywords: Multistage stochastic programming, measures of information.

\section{Introduction}

The most well known measure in two-stage stochastic programming is given by the Value of the Stochastic Solution, VSS, [2],

$$
V S S=E E V-R P
$$

where $E E V$ denotes the solution value of the stochastic programming model $R P$ where all the first stage decision variables are fixed at the optimal values obtained by using the expected value of coefficients $E V$. VSS indicates the expected gain from solving a stochastic model rather than its deterministic counterpart, when the random parameters are replaced by their expected values. A large VSS means that uncertainty greatly affects the optimal solution, and the deterministic solution is "bad". Bounds on VSS were introduced in [2] by means of the Sum of Pairs Expected Values Solutions, SPEV and Expectation of Pairs Expected Value, EPEV which can be calculated by solving pairs of simpler problems which are much less complex than the general recourse problem; these bounds may be valuable in determining whether the additional computational complexity of the recourse problem is warranted. These bounds are referred in many papers (see for instance [1], [2], [3], [4], [5], [6], [7], [8], [9], [10], [11], [12] or [13]).

In real world problems, even when the VSS is high - hence justifying the stochastic program formulation - it may be that the large size makes the problem difficult to solve. In such cases, a qualitative understanding of the deterministic solution becomes then important because it could actually lead to a lot of information: in [14] the structure and upgradeability of the deterministic solution has been analyzed in the two-stage case by means of the Loss Using the Skeleton Solution LUSS and the Loss of Upgrading the Deterministic Solution LUDS in relation to the standard VSS. Compared to the VSS, LUSS and LUDS give broader information on the structure of the problem and could be of practical relevance to take a fast "good" decision instead of using expensive direct techniques.

The aim of the paper is to present a brief summary in the multistage case of the measures of information already valid for the two-stage case introduced in $[15,14]$ and inspired in $[3,16,17]$.

An extension to multistage of the classical VSS defined for the two-stage setting, has been already introduced in [16] through a chain of values $V S S^{t}$ which takes into account the information until stage $t$ of the associated deterministic model and are valid if, in the formulation of the multistage problem, only when the right hand side of constraints are stochastic. Among the simplified approaches we mention also the Two-Stage Relaxation Problem, TP [3], where the stochasticity is taken into account only at the first stage.

\footnotetext{
a) The work has been supported under grant by Regione Lombardia: "Metodi di integrazione delle fonti energetiche rinnovabili e monitoraggio satellitare dell'impatto ambientale", EN-17, ID 17369.10 and by Bergamo and Brescia University grants 20102011.
} 
The general idea behind construction of bounds we propose, is that for every optimization problem of minimization type, lower bounds to the optimal solution can be found by relaxation of constraints and upper bound to the optimal solution can be found by inserting feasible solutions.

We suggest how to quantify the quality of approximations of the optimal stochastic solution such as the Multistage Expected Value of the Reference Scenario, MEVRS, the Multistage Sum of Pairs Expected Values, MSPEV, and the Multistage Expectation of Pairs Expected Value, MEPEV. They are introduced by means of the new concept of auxiliary scenario and redefinition of pairs subproblem probability. The proposed approaches allow to bound the optimal expected objective function by solving sets of pairs subproblems that are less complex than the original one.

Beside the standard Value of Stochastic Solution, $\mathrm{VSS}^{t}$ at stage t, we refer to [22] for measures of the average solution quality such as the Multistage Loss Using Skeleton Solution, MLUSS ${ }^{t}$ and the Multistage Loss of Upgrading the Deterministic Solution, MLUDS ${ }^{t}$ by respectively comparing the Multistage Expected Skeleton Solution Value MESSV ${ }^{t}$ and Multistage Expected Input Value MEIV ${ }^{t}$ with RP. These measures are important in practice, since they give a mathematical formulation to some heuristic approaches which are of interest in solving real world problems of large dimension.

An alternative approach here presented is to consider a rolling time horizon procedure (see [3, 17]) in order to update the estimations of the solution at each stage taking into account the arrival of new information. The advantage of the rolling horizon procedure in the multistage case with respect to the solution of the $R P$ problem, is the decrease of the computational complexity since the number of scenarios is reduced by considering the stochasticity only one stage at a time. On this subject, the Rolling Horizon Value of Stochastic Solution, RHVSS, the Rolling Horizon Loss Using Skeleton Solution, RHLUSS and the Rolling Horizon Loss of Upgrading the Deterministic Solution, RHLUDS are presented in [22] by respectively comparing the Rolling Horizon Value of the Reference Scenario RHVRS, the Rolling Horizon Skeleton Solution Value RHESSV and the Rolling Horizon Expected Input Value RHEIV with RP.

The chains of inequalities among the values of the new measures can show if and how much it is worth to face the computational complexity of the stochastic program. An example of such chains of relationships is depicted in Fig. 4 for a practical logistic problem [18].

For a more detailed presentations of the measures described shortly above, see [22].

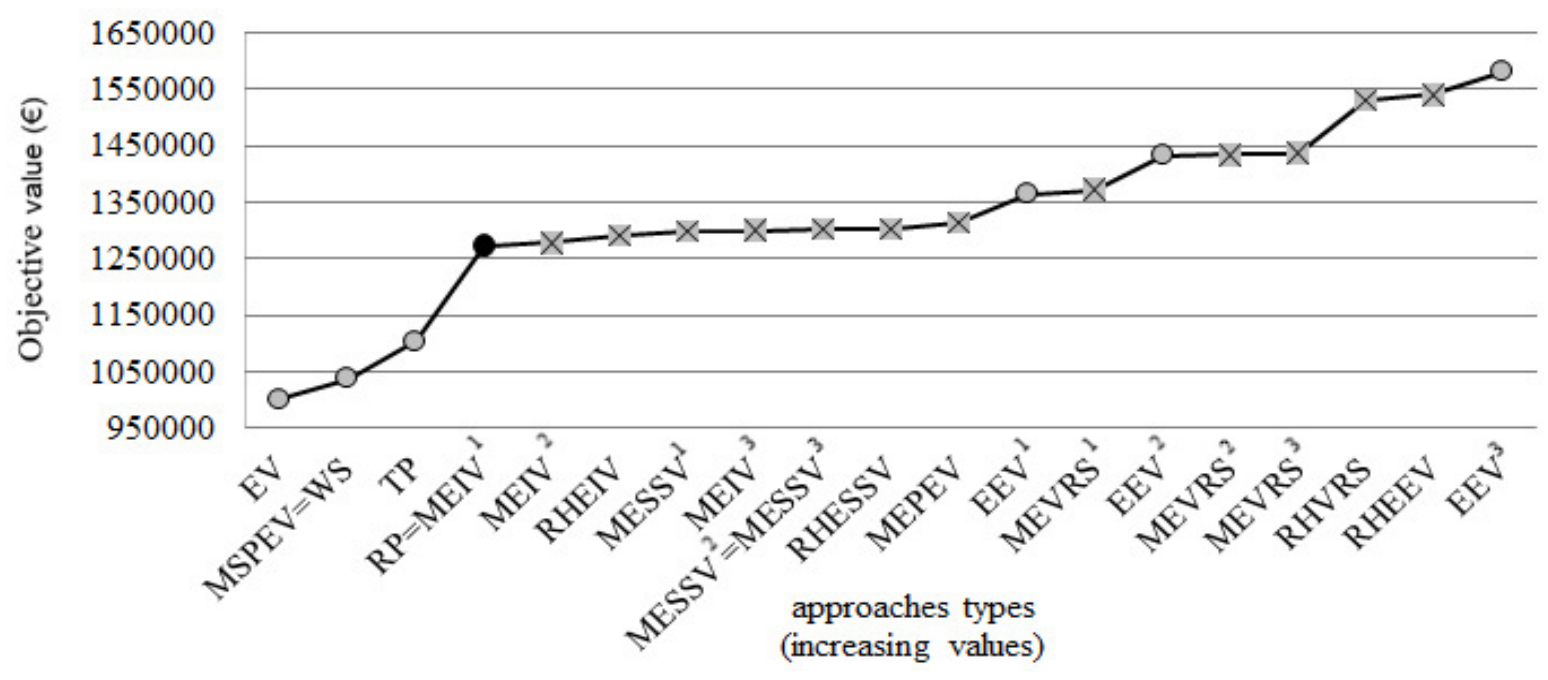

Fig. 1. Comparison of objective functions of different approaches, reported for increasing values. The black circle denotes the multistage stochastic recourse problem $R P$, the squares the values of the new performance measures introduced in [22] and the grey circles are the values of measures already known in literature

\section{Performance Measures in Multistage Problems}

In this section we discuss some of the performance measures for multistage stochastic linear problems mentioned in the introduction. Our problem can be formulated as We have to determine a sequence of decisions $\boldsymbol{x}=\left(x^{l}, x^{2}, \ldots, x^{H}\right)$ in order to minimize the linear expected costs. The optimal value obtained in correspondence of the optimal decision values is known as $R P$ (recourse problem) value. We focus on 
measures of information, where the same problem is solved and compared with and without a piece of available information on the future, and on rolling horizon measures which update the solution estimate and add more information at each stage.

\subsection{Measures of Information in Multistage Problems}

We generalize some measures introduced in [20] for the deterministic solution of the modified wait and see $(W S)$ approach and those $\left(V S S^{t}\right)$ described in [15] for the stochastic two-stage $(T=2)$ case. We consider a simplified version of the stochastic program, where only the right hand side is stochastic.

Instead of using a scenario given by the expected values of the random parameters, one may choose a specific realization $\xi_{r}, r=1, \ldots, S$, of the random variable, called the reference scenario, and solve problem $R P$. In order to extend to the multistage case the measures already available for the two stage $S P E V$ we introduce the notion of auxiliary scenario $a$ with the following characteristics:

1. $\xi_{a}=\xi_{r}$, i.e. the values of the random parameters of the auxiliary scenario are the same as the values of the random parameters of the reference scenario;

2. Let be $\hat{H}$ the first stage where the scenarios $r$ and $k$ start to branch. Define

$$
\pi_{j_{t}, j_{t+1}}=\left\{\begin{array}{cc}
\pi_{r} & \text { if } t=\hat{H} \\
1 & \text { if } t \neq \hat{H}
\end{array}\right.
$$

3. $\pi_{a}=\pi_{r}$, since $\pi_{a}=1 \cdot 1 \cdot 1 \cdot \ldots \cdot \pi_{r} \cdot \ldots \cdot 1$ bilities.

Notice that the auxiliary scenario $a$ has the same parameters values of scenario $r$ but different proba-

Let us define the PAIR subproblem as follows:

$$
\begin{gathered}
\min z^{P}\left(\boldsymbol{x}, \xi_{a}, \xi_{k}\right)=\min _{x}\left(c^{1} x^{1}+\sum_{t=2}^{\hat{H}-1} c^{t} x_{a}^{t}\left(\xi_{a}\right)+\sum_{t=H}^{H}\left[\pi_{a} c^{t} x_{a}^{t}\left(\xi_{a}\right)+\left(1-\pi_{r}\right) c^{t} x_{k}^{t}\left(\xi_{k}\right)\right]\right. \\
A x^{1}=h^{1} \\
T_{a}^{t-1} x_{a}^{t-1}+W_{a}^{t} x_{a}^{t}=h_{a}^{t} \\
T_{k}^{t-1} x_{k}^{t-1}+W_{k}^{t} x_{k}^{t}=h_{k}^{t} \\
x^{1} \geq 0, \quad x_{a}^{t} \geq 0, x_{k}^{t} \geq 0 \mathrm{t}=2, \ldots, H
\end{gathered}
$$

The Multistage Sum of Pairs Expected Values, denoted by MSPEV, is then defined as follows:

$$
M S P E V=\frac{1}{1-\pi_{a}} \sum_{k=1, k \neq r}^{n_{H}} \pi_{k} \min z^{P}\left(\boldsymbol{x}, \xi_{a}, \xi_{k}\right)
$$

The following relationships hold true (see [22]):

Proposition 2.1 If the scenario $\xi_{r}$ is not in the support of the probability space of the random variables, then $M S P E V=W S$.

Proposition 2.2 WS $\leq M S P E V$.

Proposition 2.3 $R P \geq M S P E V$.

Proposition 2.4 MEVRS ${ }^{+1} \geq M E V R S^{t}, t=1, \ldots, H-2$ where MEVRS , the Multistage Expected Value of the Reference Scenario, is the optimal value of the stochastic problem by fixing the decision variables until stage $t$ to the mean value solution of the deterministic problem under scenario $r$.

Proposition 2.5 RP $\leq M E P E V \leq M E V R S^{l}$ where $M E P E V$, the Multistage Expectation of Pairs Expected Value, is the minimum value of the optimal values of the stochastic problem obtained by fixing the first decision variables to the optimal solution of the pair subproblem of scenarios $\xi_{a}$ and $\xi_{k}$ with $k=1, \ldots, n_{H} \cap\{r\}$. 


\section{Proposition 2.6}

$0 \leq$ MEVRS $^{t}-$ MEPEV $\leq$ MVSS $^{t} \leq$ MEVRS $^{t}-M_{\text {MPEV }} \leq$ MEVRS $^{t}-\mathrm{WS}, \mathrm{t}=1, \ldots, \mathrm{H}-1$, where MVSS $^{t}=$ MEVRS $^{t}-$ RP.

In conclusion, the new measures $M S P E V$ and $M E P E V$ give respectively lower and upper bounds on the value of $R P$ using the PAIR subproblem approach. The proof of all the Propositions can be found in [22].

\subsection{Rolling Horizon Measures in Multistage Problems}

Multistage problems such as $\operatorname{MEVRS}^{t}(t=1, \ldots, H-1)$ are often infeasible since they require to fix many variables to values obtained via the expected value or the reference scenario models.

An alternative approach is to consider a rolling time horizon procedure (see $[3,17])$ in order to update the estimations of the solution at each stage taking into account the arrival of new information. Moreover, the advantage of the rolling horizon procedure in the multistage case with respect to the solution of the $R P$ problem, is the decrease of the computational complexity since the number of scenarios is reduced by considering the stochasticity only one stage at a time. We propose the following methodology for the evaluation of the reference scenario model, see [18, 21]:

1. Solve the reference scenario $r$ model and store the first stage decision variables $\vec{x}_{r}^{1}$

2. Define a new scenario tree $\mathfrak{J}^{2, e v}$ where all random parameters in stages $2, \ldots, H-1$ are replaced by their expected values and solve the related model with $x^{1}=\breve{x}_{r}^{1}$. Store all the second stage variables $\breve{x}_{r, e v}^{2}$.

3. At stage $t(t=2, \ldots, H-1)$ define a new scenario tree $\mathfrak{I}^{t+1, e v}$ where all random parameters in stages $t+1, \ldots, H-1$ are replaced with their expected value. Solve the associated model with $x^{t}=\breve{x}_{r, e v}^{t}$. Store all the $t+1$ stage variables $\breve{x}_{r, e v}^{t+1}$.

4. Finally, solve the model on the initial scenario tree with all the $t^{\text {th }}$ variables $(t=1,2, \ldots, H-1)$ fixed to the stored values $\boldsymbol{x}^{H-1}=\overrightarrow{\boldsymbol{x}}_{r, e v}^{H-1}$.

So, the Rolling Horizon Value of the Reference Scenario can be expressed as:

$$
R H V R S=E_{\xi^{H-1}} \min _{x^{H}} z\left(\overrightarrow{\boldsymbol{x}}_{r, e v}^{H-1}, x^{H}, \boldsymbol{\xi}^{H-1}\right)
$$

and the Rolling Horizon Value of Stochastic Solution with respect to reference scenario is expressed as:

$$
\text { RHVSS := RHVRS }- \text { RP. }
$$

In similar way the Rolling Horizon Loss Using Skeleton Solution RHLUSS and Rolling Horizon Loss of Upgreading the Deterministic Solution RHLUDS are defined in [22].

\section{Conclusions}

The paper extends classical measures to evaluate different approaches and levels of information from two-stage stochastic problem to the multistage case. Such generalization is both of theoretical and practical importance. The practical importance arises when solving problems of large instances where it is fundamental to have approximations of the optimal solution. The proposed methodologies have been implemented and tested, and resulted to be computationally less expensive than the full stochastic program. Indeed, we generalize bounds of Value of Stochastic Solution, VSS, to the multistage case through the Multistage Sum of Pairs of Expected Value, MSPEV, and Multistage Expectation of Pairs Expected Value, $M E P E V$, by solving a series of sub-problems computationally more tractable than the initial one, under the assumption that some additional information on the future development of a random variable is available. This extension has been done by introducing the new concept of auxiliary scenario and by the redefinition of probability of pairs subproblem. The results show that a better alternative than choosing the deterministic solution is given by the best pair subproblem solution as measured by MEPEV in case we have more information about the future. 
The above measures are also defined in a rolling horizon framework by means of the Rolling Horizon Value of Stochastic Solution, RHVVS. In these rolling horizon approaches, we update the estimations of the solution at each stage taking into account the availability of new information. The computation complexity decreases since the number of scenarios is reduced by considering the stochasticity only one stage at a time.

\section{References}

1. Avriel, M.; Williams, A. C. (1970). "The value of information and stochastic programming", Operations Research 18: 947-954. http://dx.doi.org/10.1287/opre.18.5.947

2. Birge, J. R. (1982). "The value of the stochastic solution in stochastic linear programs with fixed recourse", Mathematical Programming 24: 314-325. http://dx.doi.org/10.1007/BF01585113

3. Č́žková, J. (2006). "Value of information in stochastic programming", Working paper, Department of Probability and Mathematical Statistics, Charles University, Prague.

4. Hartley, R. (1980). Inequalities for a class of sequential stochastic decision processes, in Dempster, M.A.H. (Ed.), Stochastic Programming, Academic Press, London, pp. 109-123.

5. Hausch, D. B.; Ziemba, W. T. (1983). "Bounds on the value of information in uncertain decision problems II", Stochastics, Vol. 10, pp. 181-217. http://dx.doi.org/10.1080/17442508308833273

6. Huang, K.; Ahmed, S. (2009). "The value of multi-stage stochastic programming in capacity planning under uncertainty", Operations Research 57(4): 893-904. http://dx.doi.org/10.1287/opre.1080.0623

7. Kall, P.; Wallace, S. W. (1994). Stochastic Programming, Wiley, Chichester.

8. Madansky, A. (1960). "Inequalities for stochastic linear programming problems", Management Science 6: 197204. http://dx.doi.org/10.1287/mnsc.6.2.197

9. Pflug, G. Ch. (2004). The value of perfect information as a risk measure, in Ermoliev, Y. and Pflug, G. Ch. (Eds.), Dynamic Stochastic Optimization, Springer-Verlag, Berlin, pp. 275-292. http://dx.doi.org/10.1007/9783-642-55884-9_14

10. Pflug, G. Ch.; Ruszczyński, A. (2005). "Measuring risk for income streams", Computational Optimization and Applications, Vol. 32, pp. 161-178. http://dx.doi.org/10.1007/s10589-005-2058-3

11. Ruszczyński, A. ; Shapiro, A. (Eds.). (2003). Stochastic programming, Series Handbooks in Operations Research and Management Science, Elsevier Science B.V., Amsterdam.

12. Shapiro, A. (2008). "Stochastic programming approach to optimization under uncertainty", Mathematical Programming 112(1), Ser. B: 183-220.

13. Shapiro, A.; Dencheva, D.; Ruszczy'nski, A. (2009). Lectures on stochastic programming: Modeling and Theory, MPS-SIAM Series on Optimization.

14. Maggioni, F.; Wallace, W. S. (2010). "Analyzing the quality of the expected value solution in stochastic programming", Annals of Operations Research doi:10.1007/s10479-010-0807-x. http://dx.doi.org/10.1007/s10479010-0807-x

15. Birge, J. R.; Louveaux, F. (1997). Introduction to stochastic programming, Springer-Verlag, New York.

16. Escudero, L. F.; Garín, A.; Merino, M.; Pérez, G. (2007). "The value of the stochastic solution in multistage problems", Top, Vol. 15, pp. 48-64. http://dx.doi.org/10.1007/s11750-007-0005-4

17. Wagner, J. M.; Berman, O. (1995). "Models for planning capacity expansion of convenience stores under uncertain demand and the value of information", Annals of Operations Research 59: 19-44. http://dx.doi.org/10.1007/BF02031742

18. Maggioni, F.; Kaut, M.; Bertazzi, L. (2009). Stochastic Optimization models for a single-sink transportation problem, Computational Management Science 6: 251-267. http://dx.doi.org/10.1007/s10287-008-0086-z

19. Raiffa, H.; Schlaifer, R. (1961). Applied statistical decision theory, Harvard Business School, Boston, MA, pp. 88-92

20. Gunderson, H. S.; Morris, J. G.; Thompson, H. E. (1978). "Stochastic programming with recourse: a modification from an applications viewpoint", Journal of the Operational Research Society 29(8): 769-778.

21. Vespucci, M. T.; Maggioni, F.; Bertocchi, M. I.; Innorta, M. (2012). "A stochastic model for the daily coordination of pumped storage hydro plants and wind power plants", Annals of Operations Research 193: 91-105. http://dx.doi.org/10.1007/s10479-010-0756-4

22. Maggioni, F.; Allevi, E.; Bertocchi, M. (2012). "The value of information in multistage linear stochastic programming", submitted to Journal of Optimization Theory and its Applications 